Ann. Sci. forest., 1978, 35 (2), 117-138.

\title{
Régénération et croissance de la partie souterraine et aérienne de Cèdres placés sous climat constant
}

\author{
A. RIEDACKER \\ avec la collaboration technique de J. F. MULLER \\ Station de Sylviculture et de Production, I.N.R.A., \\ Centre National de Recherches Forestières, \\ Champenoux, 54280 Seichamps
}

\begin{abstract}
Résumé
Chaque mois deux lots de Cedrus atlantica (Manetti) élevés en pépinière ont été placés en minirhizotrons et transférés en chambre climatisée $\left(20^{\circ} \mathrm{C}\right.$ - Jour long). Toutes les racines du lot $\mathrm{T}$ (témoin) ont été conservées intactes ; celles du lot $C$ (coupé) ont été coupées à $20 \mathrm{~cm}$ du collet et privées de toutes les extrémités blanches. Nous avons observé simultanément chaque semaine le débourrement, l'allongement aérien, la régénération et l'allongement des racines, ainsi que la mortalité des plants afin de préciser notamment les périodes optimales d'arrachage des plants. Lorsque la partie aérienne est «dormante " (sept.-oct.), les racines peuvent s'allonger fortement, mais se régénèrent difficilement ; la mortalité des plants (C) est alors importante, et leur débourrement, lorsqu'ils survivent, fortement retardé par rapport aux témoins. En décembre et janvier, les plants des lots $T$ et $C$ débourrent également vite, les racines des plants $C$ se régénèrent vite et abondamment et la mortalité au repiquage est nulle.

Au moment du débourrement (fin février) et de la première vague de croissance aérienne, la régénération et l'allongement des racines se réduisent fortement ce qui se traduit par une faible mortalité des plants (C). Les vagues de croissance aérienne sont précédées et suivies par des vagues de croissance des racines, mais accompagnées par une dépression de l'allongement et de la régénération des racines, ce qui peut accentuer la mortalité à la transplantation.
\end{abstract}

Ces résultats suggèrent :

1) Sur le plan pratique :

- de s'abstenir de repiquer des Cèdres en début d'automne (sept.-oct.) ;

- de transplanter à partir de décembre ; le début de la période favorable à l'arrachage peut être détecté par les praticiens en mettant des plants, arrachés à intervalles réguliers, en minirhizotrons.

Des études in situ en cours complèteront ces données.

2) sur le plan fondamental :

- que l'évolution de l'aptitude au débourrement des plants peut dépendre, en automne, de la présence de racines blanches;

- que l'aptitude des racines à se régénérer dépend de l'état des bourgeons, et qu'en modifiant le rythme de leur évolution on doit pouvoir modifier le début des dates favorables à la régénération des racines ef donc à la transplantation. 


\section{Avant-propos}

Le Cèdre couvre déjà 10000 ha en France ; mais 120000 ha au moins de forêt méditerranéenne pourraient avantageusement être reboisés avec cette essence (Pardé, 1976).

Cependant, étant donné la reprise généralement médiocre (taux de reprise souvent inférieur à 50 p. 100) du Cèdre reboisé à racines nues, les praticiens préfèrent utiliser des plants en godets. Cette méthode est onéreuse, et dans certains cas, surtout lorsque les plants ont été élevés en sachet de polyéthylène, peut entraîner la formation de chignons, dont les conséquences fâcheuses risquent de n'apparaître que bien longtemps après la plantation (Ferrand, 1975 ; Franclet, 1975).

Bien que nous ayons pu mettre au point récemment un conteneur n'induisant plus la spiralisation des racines, le coût élevé de la production de plants en conteneurs nous conduit malgré tout à essayer d'améliorer les méthodes de plantation à racines nues.

C'est dans ce but que nous avons entrepris l'étude des variations saisonnières de la croissance et de la régénération des racines, d'une part après transfert dans des conditions constantes (chambre climatisée) et d'autre part après plantation dans deux stations aux climats bien différents (Avignon et sommet du Lubéron). Dans la présente étude il n'est fait éłat que des résultats obtenus après transfert en chambre climatisée : ils devront naturellement être confrontés par la suite à ceux de la seconde étude encore en cours. Nous ne ferons pas ici le point des connaissances sur les rythmes de croissance et de régénération des racines, une étude bibliographique sur ce sujet ayant été publiée récemment (Riedacker, 1976)

\section{Méthodologie}

Pour étudier les variations du potentiel de survie, d'allongement et de régénération des racines de plants de Cedrus aîlantica (Manetti), nous avons prélevé mensuellement deux lots $(T)$ et $(C)$ de six plants chacun issus d'un même semis et élevés sous le climat de la pépinière du C. N. R. F. (Champenoux) en sachet de polyéthylène de $50 \mathrm{~cm}$ de long dans un mélange de sable $(2 / 3)$ et de tourbe $(1 / 3)$. Les plants avaient eu deux saisons de végétation au début de l'expérience en novembre 1974.

Lot $(T)$

Les plants de ce lot ont été extraits de leurs sachets avec le maximum de précautions afin de ne pas léser les extrémités blanches des racines susceptibles a priori de s'allonger ; ils ont été placés par deux dans des minirhizotrons de $70 \mathrm{~cm}$ de haut, $30 \mathrm{~cm}$ de large (Riedacker, 1974), contenant de la tourbe.

\section{Lot $(C)$}

Les systèmes racinaires des plants de ce lot ont été sectionnés à $20 \mathrm{~cm}$ du collet, et privés de toutes les extrémités plus ou moins blanches sur la partie restante à l'aide d'une paire de ciseaux, puis placés par six en minirhizotrons identiques aux précédents, mais de $30 \mathrm{~cm}$ de haut et $70 \mathrm{~cm}$ de large.

Les deux lots ont alors été placés en chambre à climat constant $\left(20^{\circ} \mathrm{C} \pm 1^{\circ} \mathrm{C}\right.$ 
- $16 \mathrm{~h}$ de lumière -2000 Lux au niveau des plants obtenus à l'aide de tubes Sylvania Grolux. $75 \pm 15$ p. 100 d'humidité — sans alternance de température).

A la mise en place, la tourbe a été saturée par une solution nutritive diluée (Coïc, 1973) et chaque semaine au moment de la lecture de la croissance des racines, les minirhizotrons ont été retrempés durant environ $5 \mathrm{mn}$ dans cette solution à température de la chambre climatisée afin de resaturer la tourbe.

\section{Observations}

Chaque semaine nous avons noté :

- la mortalité des plants (perte de la totalité des aiguilles par contact physique),

- le débourrement,

- l'allongement des pousses aériennes,

- l'allongement des extrémités des racines (Lot $\mathrm{T}$ ) sur calque transparent à l'aide de crayons marqueurs de couleur indélébile (une couleur différente par semaine),

- la régénération ef l'allongement de nouvelles racines (Lots $T$ et $C$ ) comme cidessus. Précisons que nous appelons régénération, l'apparition de nouvelles extrémités blanches préformées ou néoformées.

La somme des allongements des racines par semaine et par plant a été obtenue à l'aide d'un curvimètre de précision à partir des relevés hebdomadaires.

\section{Résultats}

a. Mortalité (fig. 1 A)

Plants (C).

Dans les conditions de l'expérience (climat modéré, bonne alimentation hydrique), on observe deux vagues de mortalité importantes ( $>50$ p. 100) pour ces plants, l'une fin juillet se terminant en août, l'autre fin octobre et se terminant en novembre ; et deux vagues de mortalité faible, l'une en février, l'autre en mai.

Plants $(T)$.

Les plants $(T)$ ne meurent généralement pas rapidement, quelle que soit la date à laquelle on les transfère en chambre climatisée. Les deux seuls cas de mortalité observés, l'un fin février, l'autre fin août, sont probablement imputables à des accidents de manipulation ayant traumatisé les extrémités de racines lors de la mise en minirhizotrons.

\section{b. Débourrement (fig. 1 B)}

Pour caractériser le débourrement des bourgeons des populations de plants ( $T$ ) et $(C)$, nous avons retenu les critères suivants :

- le nombre total de bourgeons $(N)$ du lot ayant débourré lorsque la courbe de débourrement cumulé atteint son plafond,

- le nombre de jours séparant l'entrée en chambre climatisée du débourrement de $N / 2$ bourgeons de la population (temps moyen de débourrement de la population), 


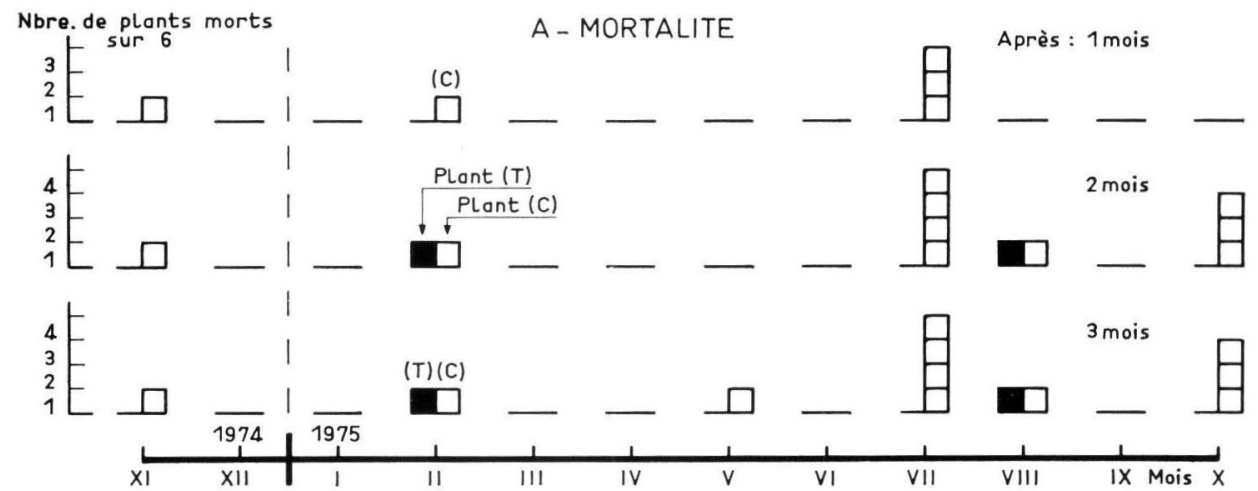

Nbre. de jours jusquóau

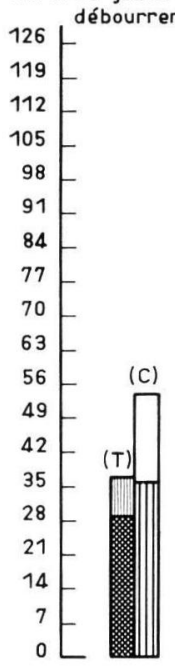

B - DEBOURREMENT

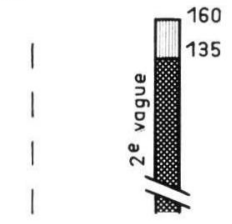

EO
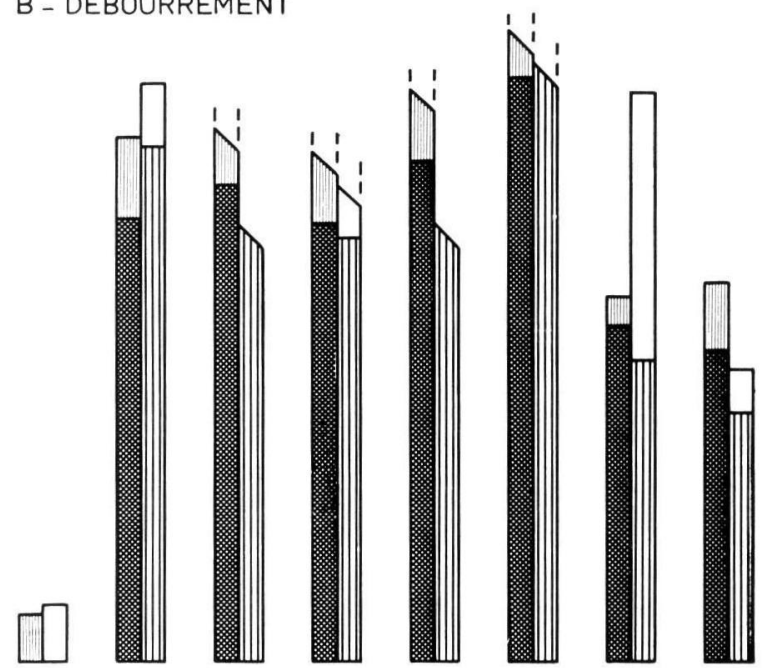

Nbre. de bourgeons
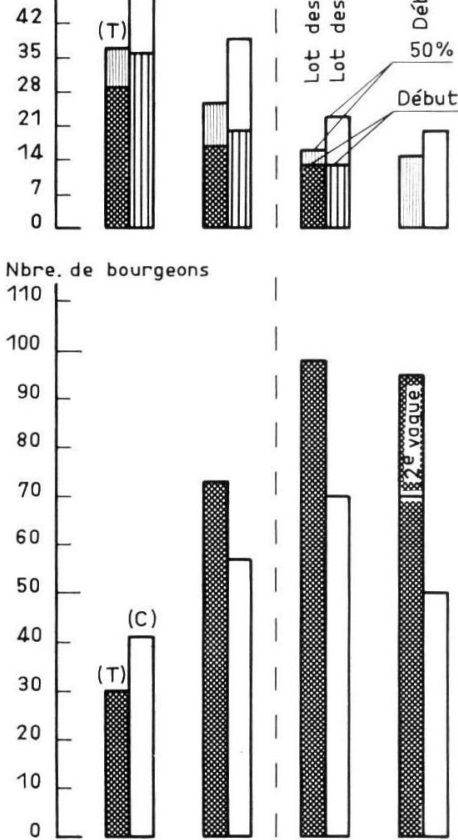<smiles>CCCCCCCCCCCCC</smiles>

EO

䓵荒

ชั้ ชั ซั

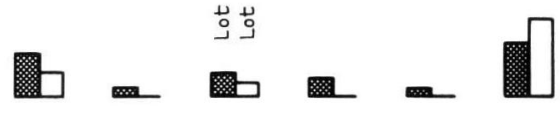
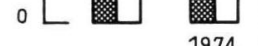

1975
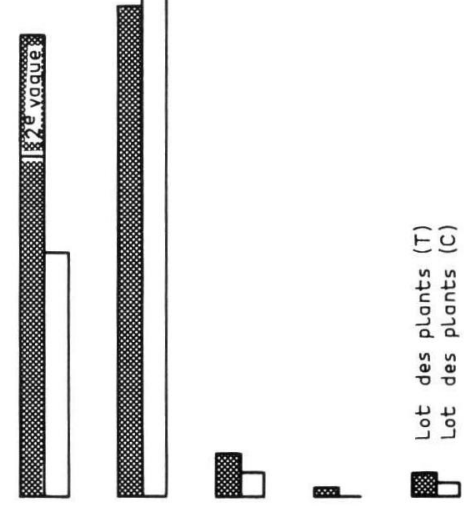
IX Mois $X$

FIG. 1, 
- le nombre de jours (L) séparant l'entrée en chambre climatisée du débourrement du premier bourgeon du lot (Temps de latence).

\section{Plants $(T)$.}

Fin avril, la quasi-totalité de bourgeons susceptible de débourrer dans la nature l'ont fait. De fin avril à fin août, il faudra plus de 3 mois après la rentrée en chambre climatisée avant que quelques rares bourgeons débourrent : un à deux par plant, et seulement sur un à deux plants par lot.

De fin septembre à fin mars le nombre de bourgeons débourrant dans chaque lot augmente plus ou moins régulièrement; fin mars une quinzaine de bourgeons débourrent en moyenne sur chaque plant. Parallèlement le temps moyen de débourrement diminue nettement à partir de novembre ; fin septembre et fin octobre il est d'environ 75 j alors que fin novembre il n'est plus que de 37 j.

Au cours de cette augmentation régulière de l'aptitude au débourrement des plants, entre novembre et mars, il semble toutefois se produire une légère dépression fin février, mais nous ne pouvons pas indiquer si elle est significative. Notons aussi que sur des plants prélevés fin février dans la nature, on observe une deuxième vague de débourrement commençant $130 \mathrm{j}$ plus tard ; une quinzaine de bourgeons apparaîtront dans ce lot.

En mars les lots n'ont malheureusement pas pu être maintenus en chambre climatisée plus de 3 mois et nous ignorons donc si une deuxième vague de débourrement aurait pu se produire.

\section{Comparaison des plants $(T)$ et $(C)$.}

En septembre le temps moyen de débourrement de plants (C) est de 42 j plus long que celui des plants $(T)$. Fin octobre, étant donné la très forte mortalité des plants (C) il est difficile de comparer les deux lots. Mais fin novembre, on observe encore un décalage de $17 \mathrm{j}$ entre les temps moyens de débourrement des deux lots.

FIG. 1 A. - Mortalité des plants 1, 2 et 3 mois après leur transfert en chambre climatisée (lots de 6 plants). (T) : Plants témoins (Noir). (C) : Plants dont les racines ont été sectionnées à $20 \mathrm{~cm}$ du collet et privés de suçoirs (Blanc).

A. - Seedling mortality 1-2 and 3 months after transfer into the growth chamber.

$\mathrm{T}=$ Control. $\mathrm{C}=$ Seedlings without white root tips and with roots cut at $20 \mathrm{~cm}$ from the collar.

\section{B. - Débourrement.}

Graphique supérieur : Nombre de jours séparant le transfert en chambre climatisée du débourrement du premier bourgeon et du débourrement de 50 p. 100 de bourgeons des lots (T) et (C).

Graphique inférieur : Nombre de bourgeons ayant débourré dans chaque lot à la fin de la vague de débourrement.

\section{B. - Budbreak.}

Above : Number of days between transfer into the growth chamber and the first budbreak, 50 p. 100 budbreak in each group of seedlings.

Underneath : Total number of buds which opened in each group of seedlings. 


\section{TABLEAU I}

Décalage $(D)$, en $j$, entre le temps moyen de débourrement des lots $(T)$ et $(C)$ Difference $(D)$, in days, of mean budbreak dates befween $(T)$ and $(C)$

\begin{tabular}{|c|c|c|}
\hline $\begin{array}{l}\text { Mise en chambre climatisée } \\
\text { (Entrance in growth chamber) }\end{array}$ & $\begin{array}{l}\text { D en jours } \\
\text { (in days) }\end{array}$ & $\begin{array}{l}\text { Seuils de signification } \\
\text { (level of significance) }\end{array}$ \\
\hline $\begin{array}{l}\text { Fin septembre } \ldots \ldots \ldots \ldots \ldots \ldots \\
\text { Fin octobre. } \ldots \ldots \ldots \ldots \ldots \ldots \ldots\end{array}$ & 42 & Non testé (Not tested) \\
\hline $\begin{array}{l}\text { Fin octobre } \ldots \ldots \ldots \ldots \ldots \ldots \\
\text { Fin novembre } \ldots \ldots \ldots \ldots \ldots \ldots \ldots\end{array}$ & $\overline{17}$ & $97 \overline{\text { p. }} 100$ \\
\hline Fin décembre $\ldots \ldots \ldots \ldots \ldots \ldots$ & 13 & 93 p. 100 \\
\hline Fin janvier............... & 7 & 93 p. 100 \\
\hline Fin février $\ldots \ldots \ldots \ldots \ldots \ldots \ldots$ & 5 & 90 p. 100 \\
\hline
\end{tabular}

Pour tester la signification de cette différence, nous avons comparé les temps moyens de débourrement pour chaque plant (50 p. 100 de bourgeons débourrés) des deux lots de plants à l'aide de l'Utest (test non paramétrique ne faisant pas d'hypothèse sur la normalité de la population des paramètres étudiés).

Le fait de couper tous les suçoirs et les racines à $20 \mathrm{~cm}$ du collet non seulement entraîne dans certains cas une forte mortalité (oct.) mais encore retarde le débourrement de fin septembre à fin décembre. La comparaison des temps de latence $L$ des lots $(T)$ et $(C)$ perm et d'aboutir à des conclusions analogues aux précédentes.

\section{c. Localisation du débourrement et de l'allongement}

Contrairement à ce que l'on peut observer chez d'autres essences forestières (Hêtre, Pin laricio) placées dans des conditions similaires, le bourgeon terminal débourre généralement le premier, et presque en même temps que les bourgeons latéraux. A partir de fin décembre les bourgeons terminaux non seulement débourrent, mais encore s'allongent (fig. 2 A).

\section{d. Mode de régénération et d'allongement des racines (fig. 2 B)}

Lorsqu'on coupe les racines à $20 \mathrm{~cm}$ du collet ainsi que toutes les extrémités blanches (Plant (C)), il apparaît d'abord une multitude de nouvelles racines (parfois une soixantaine par plant) sur tout le système racinaire. Sur les plants $(T)$, ce sont généralement d'abord les extrémités blanches préexistantes qui s'allongent; mais peu de temps après pourront naître comme sur les plants $(C)$ un nombre non négligeable de nouvelles racines; dans les deux cas seulement un petit nombre d'entre elles, celles situées sur la partie la plus éloignée du collet, continueront de s'allonger.

e. Croissance aérienne, croissance ef régénération des racines des plants $(C)$ après transfert en chambre climatisée (fig. 3)

Sur la figure 3 nous avons représenté le débourrement ainsi que la croissance aérienne et souterraine du plant moyen de chaque lot de plants (C). Cette représentation nous a paru plus satisfaisante qu'une courbe moyenne des croissances aériennes et souterraines qui, du fait de la non-synchronisation des débourrements, surtout en novembre, et en février, fait disparaître des informations intéressantes. 


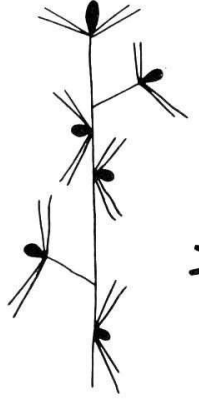

OCTOBRE

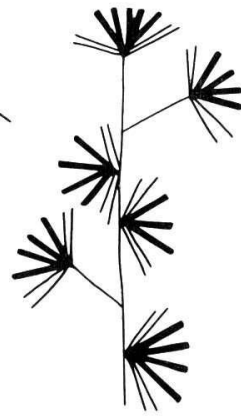

JUIN

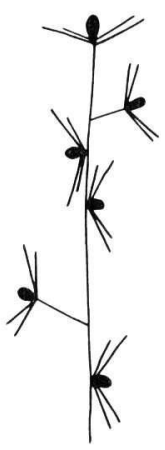

FEVRIER

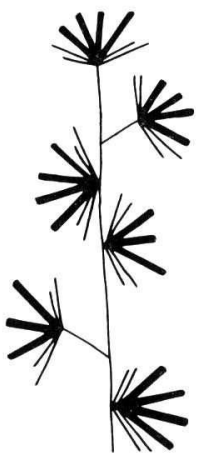

Nourelle pousse

FIG. 2 A. - Débourrement et allongement des pousses de semis de Cèdre mis en chambre climatisée en octobre et en février. Notez l'allongement du bourgeon terminal et sub-terminal des plants rentrés en chambre climatisée en février.

A. - Budbreak and shoot growth of Cedrus seedlings after transfer into growth chamber either in October or in February. Notice extension of the terminal bud when seedlings are transfered in February.
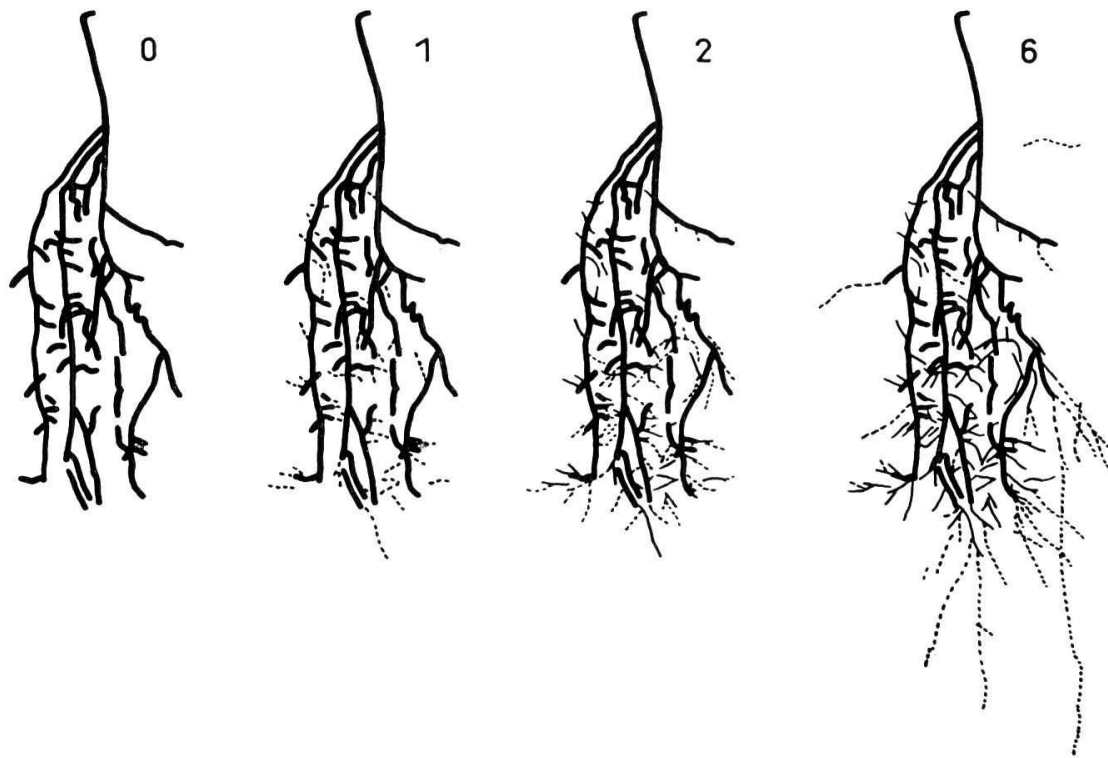

2 B. - Régénération du système racinaire d'un plant (C). 0 : Plant au début de l'expérience ; 1 : après 1 semaine ; 2 : après 2 semaines ; 6 : après 6 semaines. (-) : système racinaire primitif. (- -) : Racines nouvellement apparues ou s'étant allongées depuis le précédent relevé. (-) : Racines apparues ou s'étant allongées depuis la rentrée en chambre climatisée et avant le précédent relevé.

B. - Root regeneration of a $C$ seedling as it can be seen in a minirhizotron. $0=$ Root system when transfered into the growth chamber. 1) After 1 week. 2) after 2 weeks. 6) after 6 weeks. (-) original root system. (- - -) roots which appeared during the precedent week. ( $\rightarrow$ ) root which appeared between the date of transfer and the week preceding the last record. 

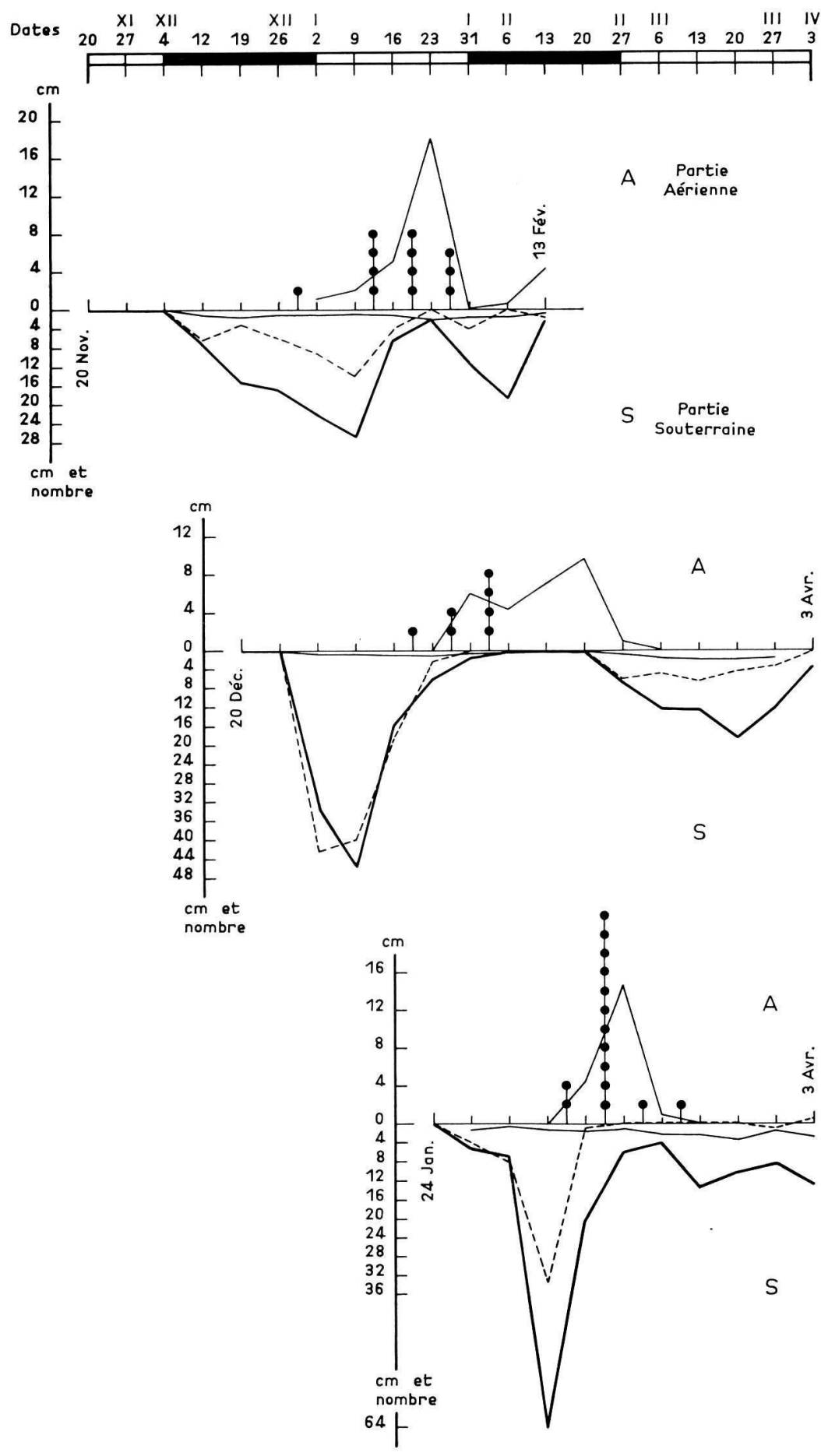

FIG. $3 \mathrm{~A}$. 

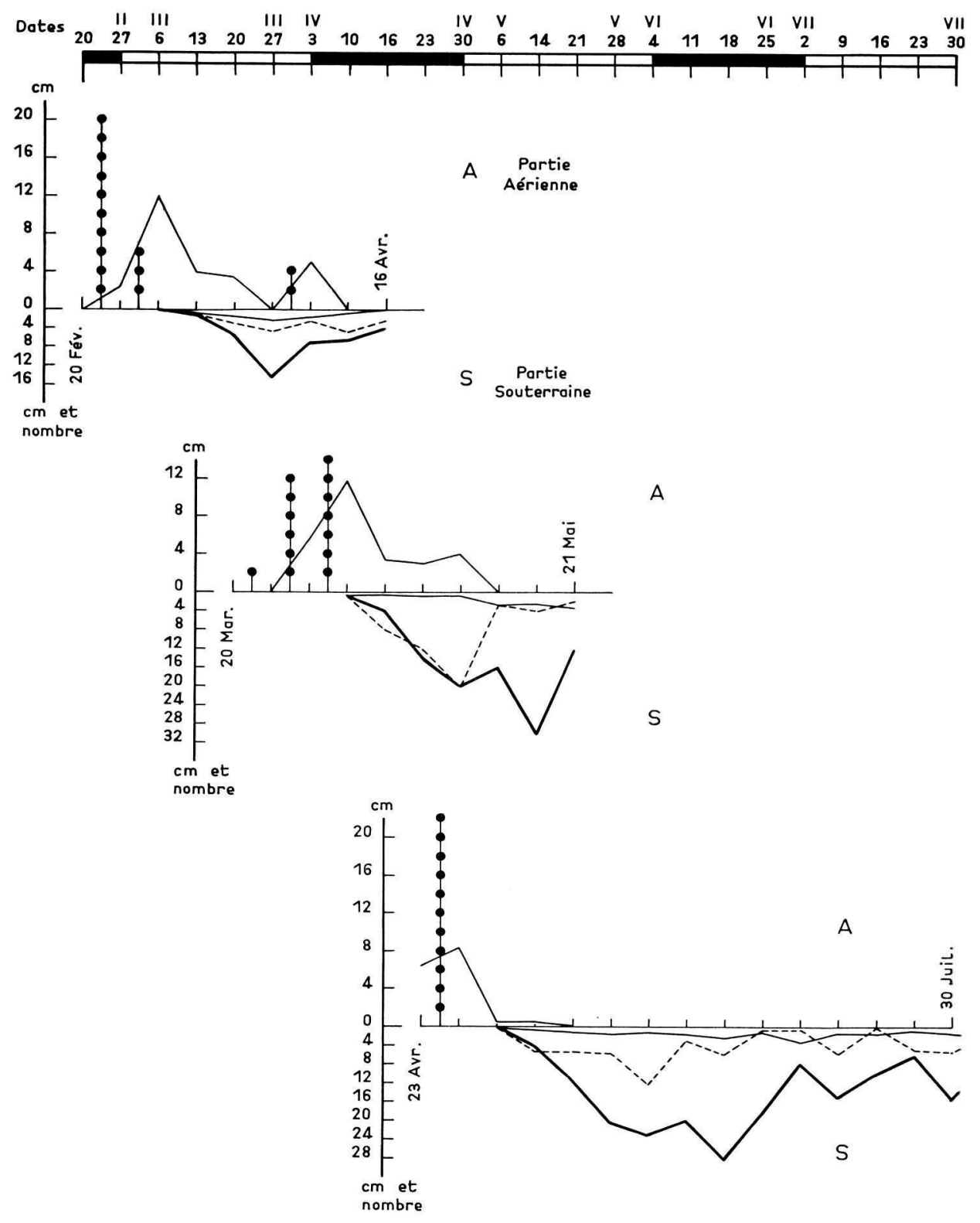

FIG. 3 B. 

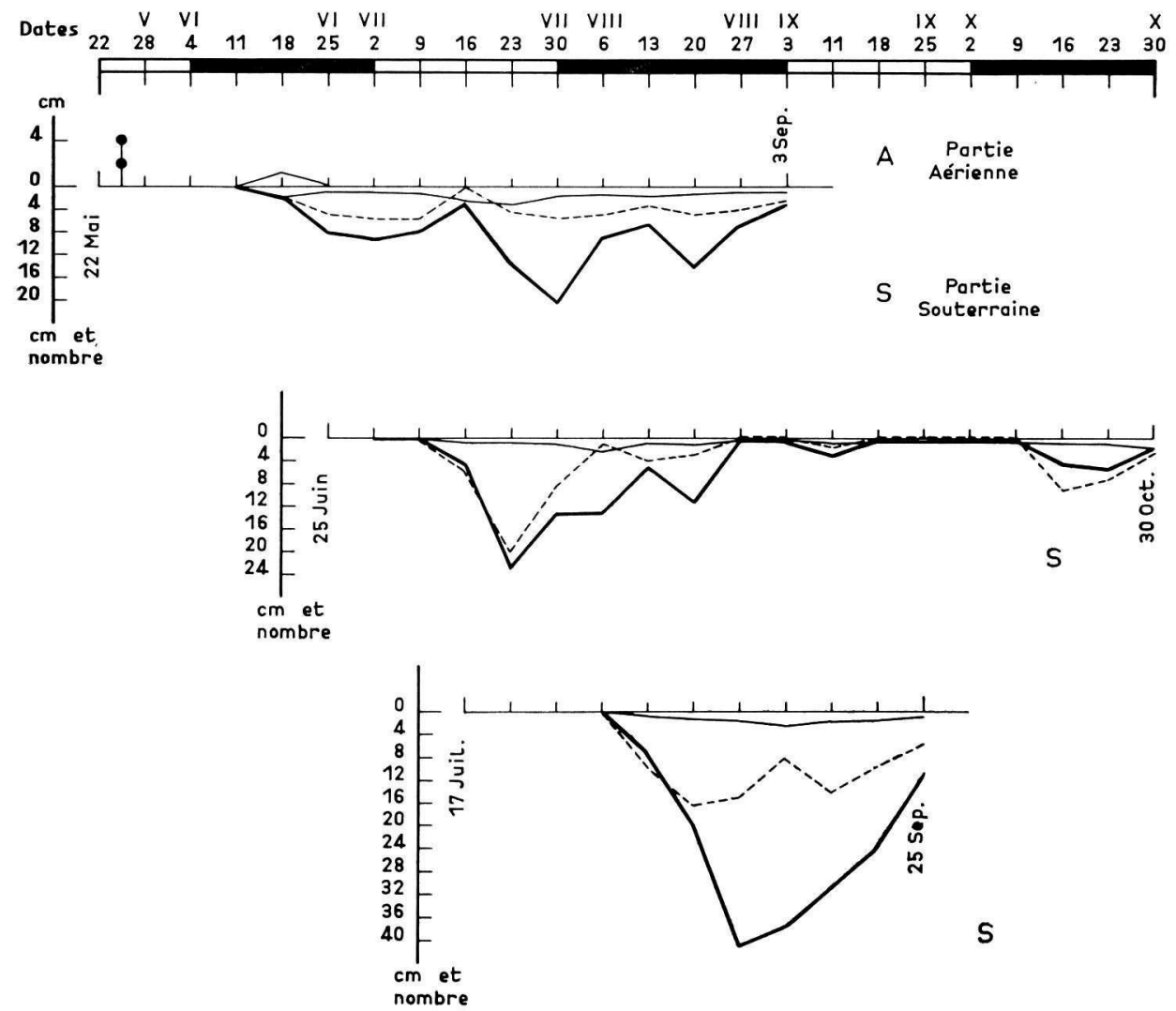

FIG. $3 \mathrm{C}$. 

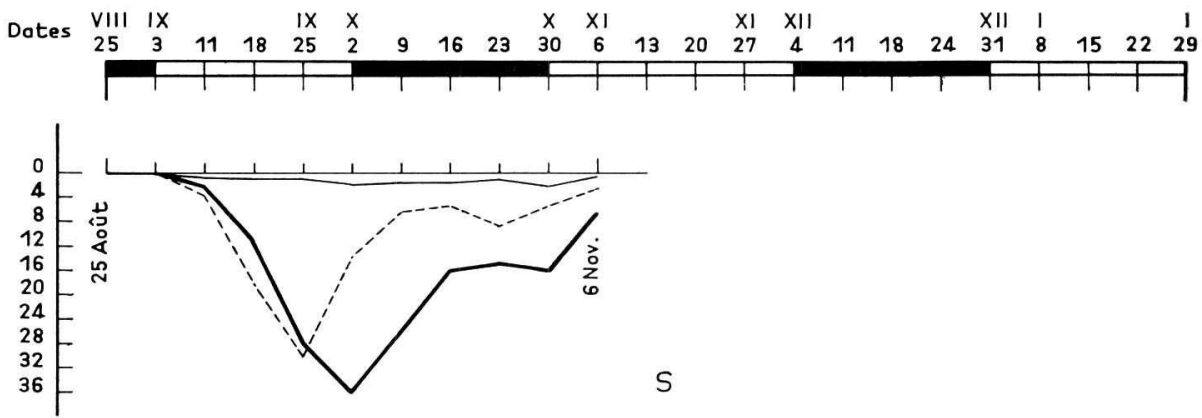

cm et

nombre
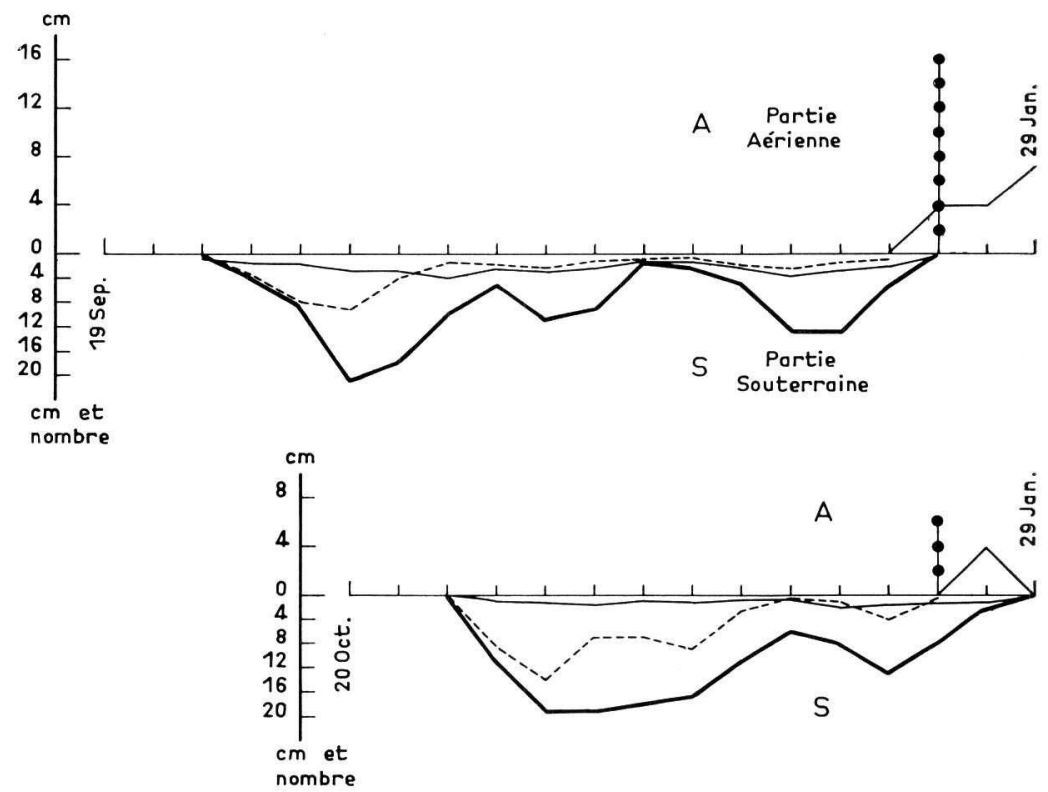

FIG. 3 D.

FIG. 3. - Diagramme de croissance des plants moyens de chaque lot de plants (C). Partie aérienne : (•) Bourgeon débourrant ; (-) Somme des allongements aériens (aiguilles + pousses) par semaine. Partie souterraine : ( - ) Somme des allongements des racines au cours de la semaine écoulée ; (- -) Nombre de racines nouvellement apparues au cours de la semaine ; (-) Vitesse moyenne d'allongement des racines par semaine. (20 nov.) : Date de transfert en chambre climatisée.
3 A. - Groupe novembre-décembre-janvier
3 B. - Groupe février-mars-avril
3 C.D - Groupe mai-juin-juillet-août-septembre-octobre.

Growth Diagram of mean $(C)$ seedlings. Shoot : $(\bullet)$ Budbreak ; (-) Total length growth of shoot + needle extension per week. Roots : (-) Total length of root extension per seedling and per week. (- - -) Number of new roots appeared per week ; (-) Mean root growth per week. (20 Nov.) Date of transfer into the growth chamber.

\footnotetext{
3 A. - November-December-January.

3 B. - February-March-April.

3 C.D - Mai-June-July-August-September-October.
} 
On peut y distinguer trois types de diagrammes; ceux de novembre-décembrejanvier, ceux de février-mars-avril, et ceux de mai à octobre.

Fin novembre à fin janvier.

Ces trois diagrammes se caractérisent par :

- une régénération et un allongement des racines important avant le débourrement et la croissance des parties aériennes ;

- l'annulation d'abord de la régénération puis de l'allongement des racines pendant la vague de croissance aérienne ;

- une seconde vague de régénération et d'allongement de racines, généralement un peu plus faible que la première, après la vague de croissance aérienne.

Fin février à fin avril.

Au cours de ces 3 mois il se produit, lorsqu'on rentre des plants en chambre climatisée, d'abord le débourrement ou la poursuite du débourrement ef l'allongement des pousses terminales; pendant ce temps les racines ne se régénèrent et ne s'allongent pas ou très faiblement. Ce n'est qu'après la cessation de la vague de croissance aérienne que l'on observe une reprise de la régénération et de la croissance des racines.

Fin mai à fin octobre.

L'allongement étant terminé fin juin, tout au moins en chambre climatisée, on n'observe alors plus qu'une croissance rythmique du système racinaire caractérisé par des vagues de régénération et d'allongement des racines suivis par des périodes de repos ou de faible activité. Les vagues qui précèdent le débourrement des plants en septembre et en octobre sont beaucoup moins importantes que celles qui précèdent le débourrement des plants rentrés à partir de la fin novembre.

\section{Remarque.}

Dès la fin octobre certains plants ont déjà un diagramme de croissance qui peut être rattaché au type « novembre, décembre, janvier », caractérisé par une vague de régénération des racines très importante précédant le débourrement; de même fin février il existe encore certains plants qui peuvent se rattacher au type « novembredécembre-janvier », c'est-à-dire présentant encore une vague de croissance racinaire avant le débourrement et la vague de croissance aérienne.

f. Croissance aérienne et souterraine des plants $(T)$ après transfert en chambre climatisée (fig. 4)

Les diagrammes de croissance aérienne et souterraine des plants $(T)$ n'ont pas une allure fondamentalement différente des plants (C). L'allongement des racines débute cependant immédiatement après le transfert en chambre climatisée grâce aux extrémités blanches préexistantes (fig. 4). Comme précédemment, aux périodes d'allongement de la partie aérienne, correspond généralement un ralentissement de l'allongement et un arrêt de la régénération des racines tandis qu'aux périodes de pré-débourrement correspond une vague de régénération des racines un peu plus importante. 


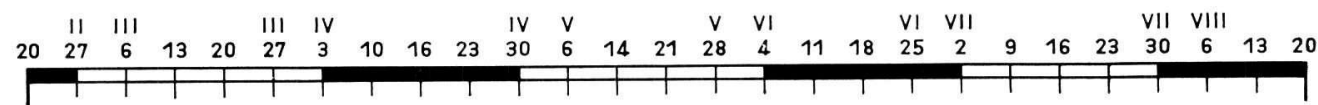

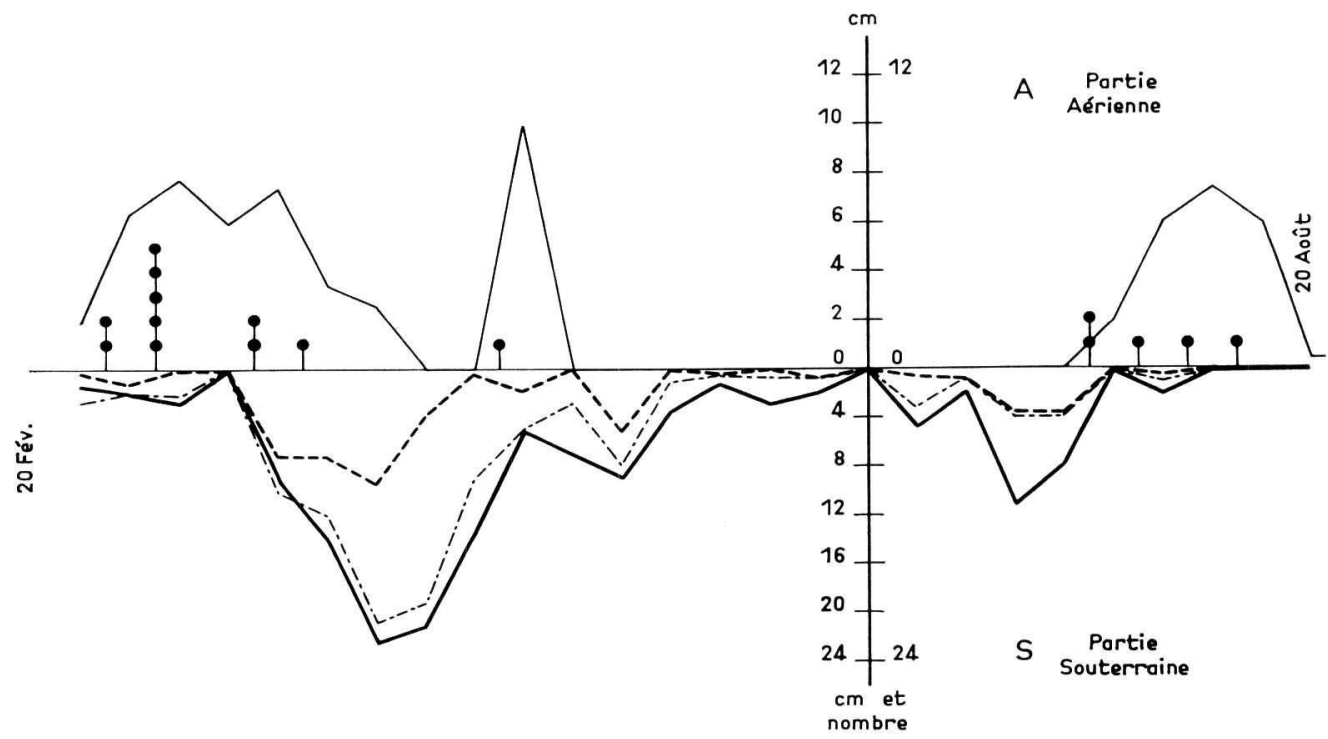

FIG. 4. - Diagramme de développement d'un plant $(T)$ après transfert en chambre climatisée le 20 février. Noter l'allongement immédiat des racines. Même légende que figure 3. (- . _ . - ) Nombre total de racines en activité.

Diagram of root and shoot growth of a $(T)$ seedling after transfer into growth chamber on the 20 th February. Notice the roots which extend without any delay.

g. L'apparition de nouvelles racines après transfert des plants en chambre climatisée (fig. 6)

Sur la figure 6 nous avons représenté le nombre cumulé de racines nouvellement apparues 1, 2, 3 et 4 semaines après le transfert des plants (T) et (C) en chambre climatisée.

Plants (C).

On note :

- un premier maximum d'apparition de nouvelles racines entre fin décembre et fin janvier (il peut en apparaître en moyenne une soixantaine par plant en 3 semaines),

- un deuxième maximum beaucoup plus faible, fin août, au cours duquel il n'apparaît en moyenne qu'environ une quinzaine de nouvelles racines par plant pendant ce même laps de temps,

- et un troisième petit maximum fin juin ne devenant visible, dans le cas présent, qu'après 4 semaines.

Ces maxima sont séparés par des périodes durant lesquelles il apparaît en moyenne en 3 semaines moins de cinq racines par plant; elles correspondent soit à un allongement actif de la partie aérienne, soit à l'entrée en « dormance ». 


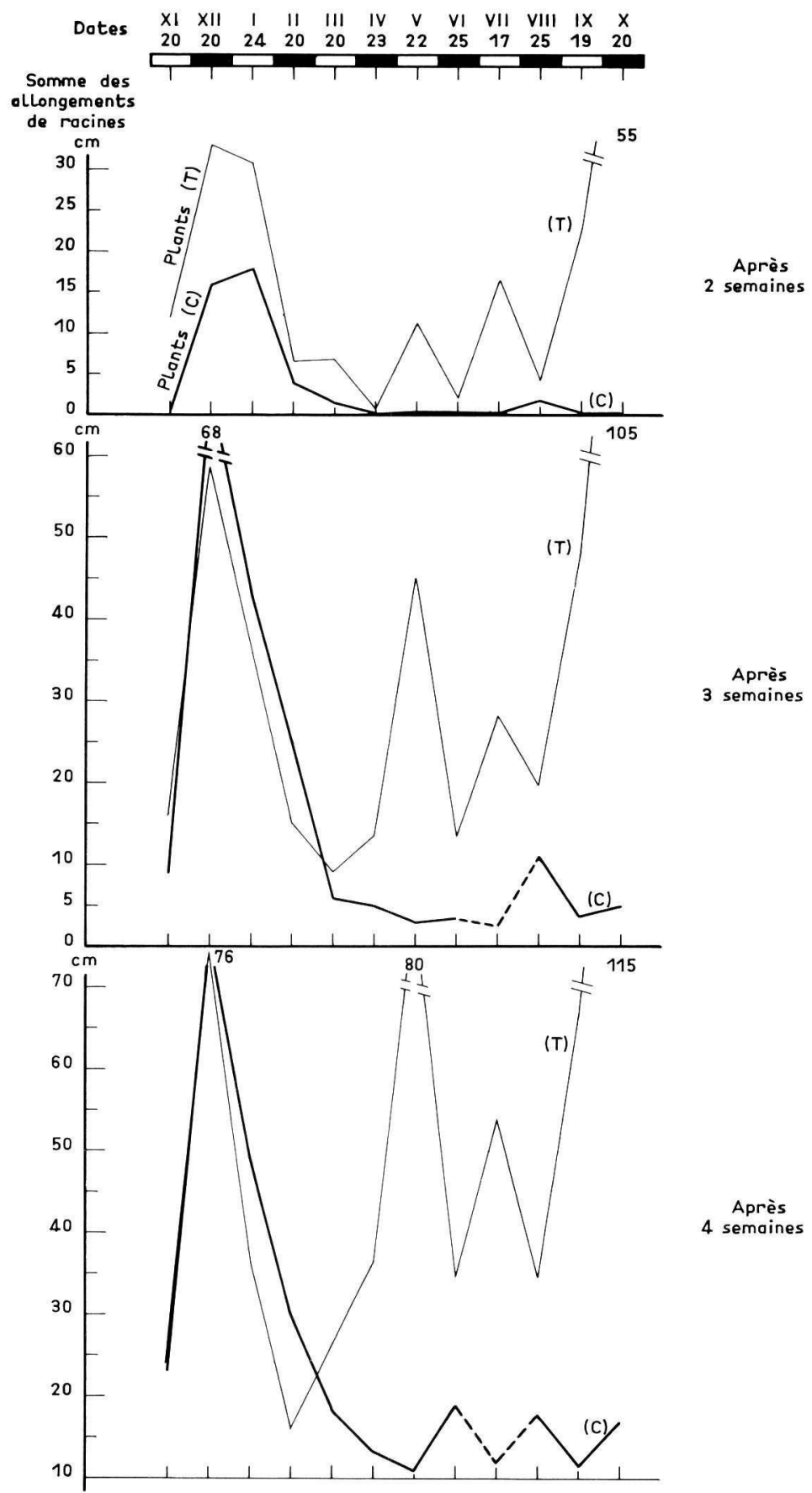

FIG. 5. - Somme moyenne des allongements des racines par plant 2, 3 et 4 semaines après le transfert en chambre climatisée ; les dates de transferts sont indiquées en haut de la figure. Les points figuratifs des plants (C) rentrés le 17 juillet sont peu précis à cause de la forte mortalité enregistrée pour ces plants.

Mean root extension per plant, 2, 3 and 4 weeks after transfer into constant conditions. Dates of transfer are indicated on top. Figurative points of (C) seedlings transfered in July are not precise because of the high mortality. 
Plants $(T)$.

En mars, au moment du débourrement, il apparaît aussi peu de nouvelles racines sur les plants $(T)$ que sur les plants $(C)$. Mais alors que la régénération des racines reste faible chez ces derniers, celles des plants $(T)$ remonte assez rapidement et reste à un niveau supérieur à dix durant tout l'été, et atteint même un niveau très élevé en septembre et octobre, au moment où les racines des plants (C) sont incapables de se régénérer.

h. L'allongement des racines après transfert des plants en chambre climatisée (fig. 5)

La figure 5 représente la somme des allongements des racines 1, 2, 3 et 4 semaines après le transfert en chambre climatisée.

Plants (C).

Les courbes obtenues diffèrent de la figure 6 par le fait que :

- le maximum d'août est moins visible,

- le maximum de juin n'est pas détectable avant 4 semaines.

Plants $(T)$.

Les diagrammes représentant l'allongement cumulé des racines des plants (T) sont caractérisables par le fait :

- qu'en décembre la somme des allongements des plants (T) est après 3 ou 4 semaines aussi importante que celle des plants (C),

- que les maxima d'allongement de mai et juillet sont nettement plus marqués que les maxima de régénération de racines aux mêmes périodes.

\section{i. Remarques sur la méthodologie}

Il apparaît d'après les diagrammes des figures 5 et 6 ainsi que d'après la figure 4 , qu'en chambre à climat constant $\left(20^{\circ} \mathrm{C}\right)$ :

- la vitesse moyenne d'allongement des racines fluctue seulement entre $0 \mathrm{~cm}$ (lorsqu'il y a arrêt) et $2,5 \mathrm{~cm}$ ( 1 à $2,5 \mathrm{~cm}$ par semaine lorsque les racines s'allongent)

- que la somme des allongements et le nombre des racines en activité sont deux indices qui varient dans le même sens.

Il est par conséquent possible dans ces conditions, lorsqu'on ne cherche pas à analyser très finement les phénomènes de prendre l'un ou l'autre des critères pour caractériser l'activité des systèmes racinaires des Cèdres.

Il apparaît également qu'une quinzaine de jours après transfert des plants en chambre climatisée on peut déjà obtenir une assez bonne image du potentiel de régénération des racines; après 3 semaines cette image ne s'améliore pratiquement plus.

Il est donc préférable, si l'on veut tester l'état physiologique des plants au moment de l'arrachage et non pas leur modification au cours du séjour en chambre climatisée de ne pas prolonger davantage le test. 


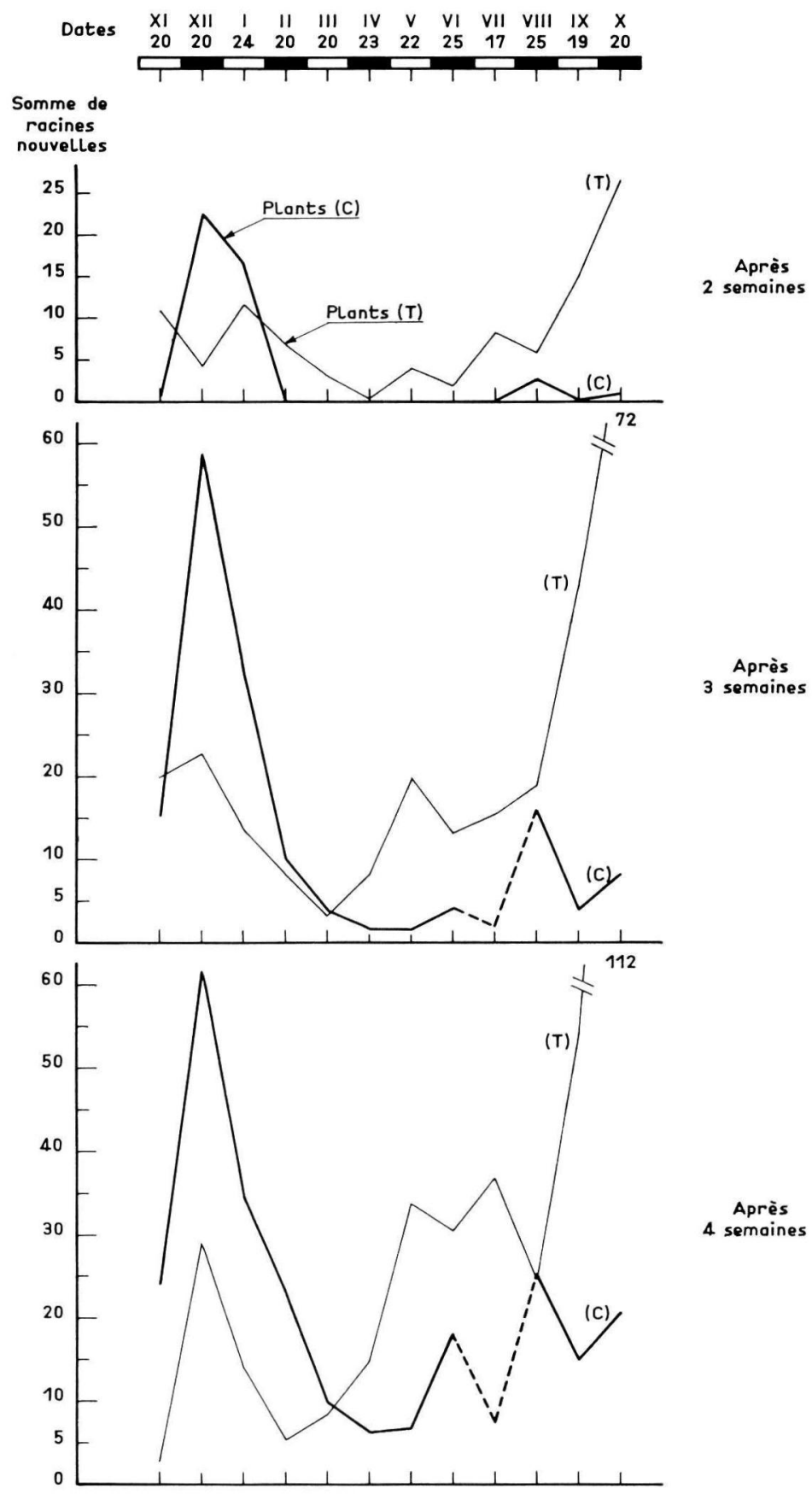

FIG. 6. - Nombre moyen de racines nouvelles par plant 2, 3 et 4 semaines après le transfert en chambre climatisée ; les dates de transferts sont indiquées en haut de la figure. Les points figuratifs des plants rentrés le 17 juillet sont peu précis à cause de la forte mortalité enregistrée pour ces plants.

Mean number of new roots per plant 2, 3, 4 weeks after transfer into growth chamber ; transfer dates are indicated on top of the diagram. Figurative points of $(C)$ seedlinqs transfered in July are not precise because of the high mortality. 


\section{Conclusions et discussion}

Les résultats de cette étude permettent dès maintenant de formuler un certain nombre de conclusions. Ils devront néanmoins être confrontés ultérieurement aux résultats de l'étude en cours sur les variations de la régénération et de la croissance des racines après transplantation en conditions naturelles.

\section{Le débourrement}

Nous n'avons pas étudié à proprement parler ici l'évolution de la dormance de bourgeons, car il eût alors fallu pour cela étudier le débourrement non pas de plantes entières, mais de boutures de nœuds (Champagnat et coll., 1975). Une telle étude n'a pas encore été tentée, à notre connaissance, avec des résineux.

Les bourgeons de Cèdre non isolés de la plante paraissent avoir besoin, pour débourrer normalement, de subir durant une certaine période, l'action du froid, puis de températures plus élevées, comme la plupart des espèces ligneuses des régions tempérées.

Mais lorsqu'on supprime toutes les extrémités blanches des racines le débourrement de ces plants est retardé en automne. II semble que les bourgeons et peut-être toute la partie aérienne aient alors besoin pour évoluer normalement, non seulement de subir une certaine période de froid, mais encore de la présence de racines blanches. Il n'est pas exclu que celles-ci fournissent des produits du type Gibberelline ; Arias et Crabbe (1975) ont en effet montré qu'une injection de Gibberelline à la base des bourgeons, ou l'alimentation de bouture de nœuds par de l'eau contenant des Gibberellines étaient susceptibles de réduire la dormance des bourgeons de Cerisier en automne.

Lavender et coll. (1973) ont aussi montré que chez des plants de Douglas, un abaissement de la température des racines $\left(5^{\circ}\right)$ pouvait retarder le débourrement d'une quinzaine de jours par rapport aux témoins ayant leurs racines à même température $\left(20^{\circ} \mathrm{C}\right)$ que la partie aérienne. Lorsque la partie aérienne est aspergée avec une solution contenant 100 ppm d'acide gibberellique les plants ayant leurs racines à $5^{\circ} \mathrm{C}$ débourrent en même temps que les témoins.

Une analyse des exsudats racinaires aurait montré que la température des racines peut influencer In teneur en produits de type Gibberellines.

Sans doute n'existe-t-il pas de relation simple entre le système racinaire et la partie aérienne ; mais peut-être, tout au moins chez certaines essences, la présence de racines actives (ce qui n'implique pas a priori qu'elles s'allongent) est-elle indispensable à l'évolution normale des bourgeons au même titre que le froid?

\section{La régénération des racines}

Pendant la période qui précède le débourrement, il apparaît un grand nombre de nouvelles racines; ce nombre augmente parallèlement à l'augmentation du nombre de bourgeons susceptibles de débourrer et à la vitesse de débourrement, mais seulement jusqu'à un certain point. En effet, lorsque le débourrement a lieu moins de $15 \mathrm{j}$ après le transfert en chambre climatisée, la régénération des racines ne peut plus avoir lieu avant la vague de croissance aérienne. C'est en décembre et janvier, alors 
qu'une dizaine de bourgeons par plant seront susceptibles de débourrer, et les pousses terminales capables de s'allonger, que l'on observe la plus importante vague de régénération des racines. Aux petites vagues de débourrement ayant lieu par la suite ne correspondent que des petires vagues de régénérarion de racines. Quelles hyporhèses peut-on avancer pour expliquer ces phénomènes?

Les bourgeons, ou toute la partie aérienne, en entrant dans la période de prédébourrement stimulent-ils alors la régénération des racines et par quel mécanisme?

Quoi qu'il en soit, si l'entrée en période de prédébourrement coincide avec une vague potentielle de régénération des racines, on devrait pouvoir modifier le rythme de régénération des racines en modifiant l'évolution de la dormance des bourgeons

Notons aussi que chez le Douglas (Lupke, 1976 et Stone et coll., 1962), à la période de prédébourrement correspond le potentiel de régénération des racines le plus élevé.

\section{Alternance de croissance aérienne et souterraine}

Lorsque les bourgeons débourrent, et que les aiguilles ainsi que parfois les pousses terminales s'allongent, la régénération des racines cesse, tandis que leur allongement se poursuit encore pendant 1 à 2 semaines. Vers la fin de la vague de croissance aérienne, quelques racines nouvelles se forment, et certaines racines recommencent à s'allonger.

Plusieurs hypothèses, déjà discutées par ailleurs (Riedacker, 1976), peuvent être avancées pour expliquer ces phénomènes:

1) Lorsque la partie aérienne croît, elle utilise prioritairement tous les glucides solubles de la plante ; lorsque ce pool de glucides (pouvant être alimenté par les réserves et la photosynthèse) est trop faible par rapport à la demande de la partie aérienne, il pourrait ne plus y en avoir suffisamment pour permettre la régénération, puis la croissance des racines.

2) Pendant la vague de croissance de la partie aérienne, la croissance et la régénération des racines sont bloquées soit par carence en certains produits essentiels (autres que les glucides) pour leur croissance et provenant habituellement de la partie aérienne, soit par l'émission de produits inhibiteurs de la croissance des racines.

L'expérimentation évoquée dans cette note ne permet pas d'avancer des arguments en faveur de l'une ou l'autre de ces deux hypothèses.

Représentation schématique de l'évolution chronologique des racines et de la partie aérienne

\begin{tabular}{ccc}
\hline $\begin{array}{c}\text { Périodes } \\
\text { approximatives }\end{array}$ & Partie aérienne & Racines \\
\hline
\end{tabular}

Dormance *

Septembre octobre
Les bourgeons (et la partie aérienne?) ont besoin de froid ET des racines pour évoluer (débourrer puis s'allonger) (Hypothèse).
Allongement potentiel des racines important mais régénération très difficile. 
Prédébourrement ou Post-dormance *

décembre janvier

Mars

avril

mai

juillet

septembre octobre
Les bourgeons sont capables de débourrer assez rapidement (et le bourgeon terminal de s'allonger) sans l'aide des racines ni du froid. La partie aérienne est capable de stimuler la régénération des racines

Débourrement ef première vague de croissance

Arrêt de croissance aérienne Période de pré-débourrement pour la deuxième vague de croissance

Deuxième vague de croissance aérienne

Arrêt de croissance aérienne

Dormance * (Voir au début)
- Le potentiel de régénération des racines est très élevé.

- L'allongement des racines est possible.

Réduction et éventuellement arrêt de la régénération des racines et de leur allongement.

Légère reprise de l'allongement et de la régénération des racines.

Réduction et éventuellement arrêt de la régénération et de l'allongement des racines.

Légère reprise de la croissance et de la régénération des racines

* Le terme «Dormance » signifie ici, absence de débourrement rapide lorsque le plant est remis dans des conditions permettant «normalement » la croissance.

Mortalité et évolution de la partie aérienne

Les conditions climatiques dans lesquelles nous avons transféré les plants sont modérées ; la mortalité observable sur des lots de plants mis en place en conditions naturelles risque donc, a priori, d'être encore beaucoup plus forte que dans le cas présent. talité :

Malgré ces conditions favorables il y a deux fortes et deux faibles vagues de mor-

- celle de juillet (avec un résidu en août) de forte mortalité qui correspond sans doute à la deuxième vague de croissance,

- celle d'octobre (avec un résidu en novembre) de forte mortalité qui correspond sans doute à la période de « dormance » maximale des bourgeons,

- celle de février de faible mortalité qui correspond sans doute au débourrement,

- celle de mai, également de faible mortalité, qui correspond sans doute à la première vague de croissance.

\section{Conséquences pratiques}

Les résultats de cette étude ne permettent pas encore de tirer des conclusions définitives.

D'après le schéma précédent, il apparaît néanmoins que :

- l'arrachage est sans doute à déconseiller fortement pendant la période de forte dormance des bourgeons c'est-à-dire en septembre-octobre ; 
- l'arrachage est possible, et sans doute conseillé en décembre-janvier car alors, d'une part la partie aérienne n'a plus besoin des racines pour évoluer et d'autre part le potentiel de régénération des racines est maximum ;

- l'arrachage est sans doute à déconseiller de fin mars à mai, car alors le débourrement et la croissance aérienne freinent la régénération et l'allongement des racines ;

- de mai à septembre on ne reboise pas, car les conditions de sol en région méditerranéenne sont généralement peu favorables.

La vague de régénération des racines que l'on peut obtenir fin août, bien que faible, serait sans doute, si l'on pouvait conserver cette potentialité jusqu'au moment des pluies d'octobre, particulièrement intéressante ; elle permettrait probablement d'obtenir une bonne installation des plants dès l'automne pendant que le sol est encore chaud.

Lupke (1976) a d'ailleurs montré que chez l'Epicéa il n'y avait pas de relations directe entre l'abondance de racines régénérées et le rétablissement d'une transpiration normale des plants; très peu de suçoirs suffiraient pour revenir à un régime hydrique normal.

Il convient donc maintenant de déterminer comment on peut tirer le meilleur parti du fort potentiel de régénération des racines en décembre-janvier, et du moins fort potentiel de régénération de la fin août et notamment de voir comment ces potentiels se conservent lorsqu'on place les plants en chambre froide, ou lorsqu'on les plante dans un sol trop froid pour l'expression immédiate de cette potentialité (en déc.).

Notons aussi, que même à la température de $20^{\circ} \mathrm{C}$ il faut attendre 8 à $10 \mathrm{j}$ avant de voir apparaître les premières régénérations de racines. La conservation, tout au moins des racines, dans un milieu chaud * et humide durant une huitaine de jours après l'arrachage ef avant plantation, pourrait donc se révéler être une pratique intéressante. Un retour éventuel ultérieur à des températures basses pour la conservation ( $8^{\circ} \mathrm{C}$ ou moins) permettrait ensuite de bloquer temporairement l'allongement des racines néoformées.

Si les études actuellement en cours sur le terrain confirment qu'il convient d'arracher les plants lorsque le potentiel de régénération des racines est élevé, il sera très facile de mettre à la disposition des praticiens un moyen permettant de déterminer le début et la fin des périodes optimales d'arrachage des plants en pépinière. On s'aperçoit en effet, d'après les diagrammes d'évolution du potentiel de régénération des racines (fig. 5), qu'une quinzaine de jours après l'installation des plants en minirhizotrons on obtient déjà une assez bonne image des potentialités du plant. Le test de Stone et coll. (1962) dont cette méthode est d'ailleurs assez proche, ne permet d'obtenir ces informations qu'au bout d'un mois. A l'approche des périodes favorables le praticien pourra donc placer en minirhizotron chaque semaine, et cela durant une quinzaine de jours six plants d'un même lot arrachés en pépinière dont on a coupé les racines à $20 \mathrm{~cm}$ ainsi que toutes les extrémités blanches. Lorsqu'il obtiendra une régénération importante au bout de ce laps de temps sur tous les plants, il pourra commencer leur arrachage. II pourra de la même manière tester l'évolution au cours du temps du potentiel de régénération de plants conservés en chambre froide.

Actuellement nous recommandons de placer les minirhizotrons à $20^{\circ} \mathrm{C}$ et $16 \mathrm{~h}$ de

* Selon Stone et coll. (1962) et Lupke (1976) le nombre de suçoirs régénérés à basse température (10-12 ou $15^{\circ}$ ) est beaucoup plus faible qu'à $20^{\circ} \mathrm{C}$. 
jour ; pour simplifier encore la méthode, nous étudions s'il est possible d'obtenir les mêmes résultats en plaçant les plants à l'obscurité et si en opérant à une température un peu plus élevée on ne pourrait pas obtenir ces informations encore plus rapidement ou de manière plus tranchée.

II n'est pas exclu qu'en appliquant ces tests dans diverses pépinières pendant 2 ou 3 ans, l'on découvre des climats permettant d'obtenir un potentiel de régénération des racines élevé moins éloigné des moments favorables pour la plantation.

Le potentiel d'allongement des racines issues de plants en mottes est élevé en mai, décembre et octobre ; par conséquent il peut être avantageux de mettre en place les plants en godet dès les premières pluies d'automne, avant que le sol ne se soit trop refroidi.

Reçu pour publication en décembre 1977.

\section{Summary \\ Root regeneration and Root and Shoot growth of Cedrus atlantica Manetti transfered into a constant climate}

Each month six seedlings $(T)$ with intact roots and six seedlings $(C)$ without white root tips or roots distant more than $20 \mathrm{~cm}$ from the collar, were transfered from the nursery into minirhizotrons in a growth chamber (20\% C longday).

Simultaneously we observed each week bud break and shoot growth, root regeneration and growth as well as plant mortality in order to find out the best planting periods.

When buds are deeply dormant (September-October) roots may extend rapidly but do not regenerate easily; seedling mortality after transplantation is correspondingly high, and bud break of the surviving seedlings delayed.

In December roots of $(T)$ and $(C)$ seedlings regenerate well and rapidly ; later on buds break almost simultaneously.

During budbreak (end of February) and the first shoot growth, root growth and regeneration are depressed and seedling mortality slightly increased.

After shoot growth, roots of surviving seedlings grow and regenerate well.

The results suggest:

1) From a practical viewpoint :

- that it is not recommended to transplant Cedrus seedling in the early autumn (SeptemberOctober) ;

- that from December on it is possible to transplant the seedlings. The beginning of this period can be precised by putting six seedlings every week into a minirhizotron during two weeks.

Field studies are underway to complete these informations.

2) From a fundamental viewpoint :

- that budbreak ability in autumn may depend on the presence of white root tips ;

- that root regeneration may depend on the evolution of buds and that by modifying the evolution of bud dormancy we should be able to modify the evolution of the root regeneration potentiel.

\section{Références bibliographiques}

ARIAS A., CRABBE J., 1975. Les gradients morphogénétiques du rameau d'un an des végétaux ligneux en repos apparent. Données complémentaires fournies par l'étude de Prunus avium L. Physiol. veg., 13 (1), 69-81.

CHAMPAGNAT P., LAVARENNE S., BARNOLA P., 1975. Corrélation entre bourgeons et intensité de la dormance sur le rameau de l'année pour quelques végétaux ligneux en repos apparent. C. R. Acad. Sci., Paris, t. 280, Série D, Pp. 2219-2222.

COIC Y., LESAINT C., 1973. La nutrition minérale en horticulturt. Revue horticole, août-sept., no 2, p. 316. 
FERRAND, 1975. Etude expérimentale et observations relatives à la morphogenèse et à la croissance du système racinaire du semis de Cèdre. Publication interne Station de Sylviculture, 45 p.

FRANCLET, 1975. Comment choisir un conteneur pour une production de plants forestiers. In Compte rendu des réunions du groupe d'étude des racines, Tome 3. Edité par A. Riedacker et J. Gagnaire-Michaud, Pp. 12-33.

LAVENDER, D. P., G. B. SWEET, J. B. ZAERR, R. K. HERMAN, 1973. Spring shoot growth in Douglas fir may be initiated by Gibberellins Exported from the roots. Science 23 nov. 1973 ; Vol. 181, pp. 838-839.

LUPKE, 1976. Wurzel regeneration von jungen Forstpflanzen nach dem Verpflanzen Forstarchiv. $\mathrm{n}: 12$, pp. $245-255$.

PARDÉ J., 1976. Il Cedro nella zona mediterranea francese. Annali dell'Accademia Italiana di Science Forestali. Firenze, Vol. XXV, pp. 269-285.

RIEDACKER, 1974. Un nouvel outil pour l'étude des racines et de la rhizosphère ; le minirhizotron. Ann. Sci. For., 31 (2), 129-134.

RIEDACKER, 1976. Rythmes de croissance et de régénération des racines des végétaux ligneux. Ann. Sci. For., 33 (3), 109-138.

STONE E. C., JENKINSON J. L., KRUGMANN S. L., 1962. Root regenerating potential of Douglas fir seedlings lifted at different times of the year. For. Sci., 8, 288-297. 